QUARTERLY OF APPLIED MATHEMATICS

VOLUME LXX, NUMBER 2

JUNE 2012, PAGES 393-405

S 0033-569X(2012)01261-1

Article electronically published on March 1, 2012

\title{
ON THE REGULARITY OF DEEP-WATER WAVES WITH GENERAL VORTICITY DISTRIBUTIONS
}

\author{
$\mathrm{BY}$ \\ BOGDAN-VASILE MATIOC \\ Institut für Angewandte Mathematik, Leibniz Universität Hannover, Welfengarten 1, 30167 Hannover, \\ Germany
}

\begin{abstract}
We prove that the streamlines and the profile of traveling deep-water waves with Hölder continuous vorticity function are smooth, provided there are no stagnation points in the flow. In addition, if the vorticity function is real analytic, then so is the profile of both solitary and periodic traveling deep-water waves. Finally, by choosing appropriate weighted Sobolev spaces, we show that the streamlines beneath the surface of a periodic traveling water wave are in fact real analytic, provided the vorticity function is merely integrable against a cubic weight.
\end{abstract}

1. Introduction. In this paper we are concerned with the regularity of the free surface and of the streamlines of gravity water waves traveling above an ocean of infinite depth in both a periodic and solitary regime. We allow only for water waves traveling at a constant speed which exceeds the horizontal speed of individual fluid particles, so that the existence of stationary points is excluded. Moreover, considering water waves with general vorticity, the classical regularity result obtained for rotational waves in [26] is a particular case of our analysis.

It was Gerstner [16] who first found a family of explicit periodic deep-water waves having a nonzero vorticity which decreases rapidly with depth; cf. [5, 21]. This behavior was confirmed in a general setting in [8] where it is shown that steady periodic deep-water waves with a monotone profile between crests and troughs, propagating against a current with a vorticity that is nonincreasing and has bounded first order partial derivatives, must be symmetric and the vorticity has to be nonpositive and vanishing with depth. Later results concerning existence of traveling water waves with general vorticity can be found in [13, small amplitude waves being obtained by using series expansion methods. The methods employed in [10] to prove existence of steady periodic water waves traveling above a flat bed and with a general vorticity function have been adapted in [31] to the infinite depth case. The author establishes therein the existence of periodic steady

Received November 17, 2010.

2010 Mathematics Subject Classification. Primary 35J25, 76B03, 76B47.

Key words and phrases. Deep-water waves, streamlines, vorticity.

E-mail address: matioc@ifam.uni-hannover.de 
deep-water waves with vorticity and having a single crest and trough per wavelength. It should be mentioned that we do not assume in this paper any kind of symmetry of the water waves we consider. Concerning existence of solitary deep-water waves we refer to [18] where existence of gravity-capillary surface waves on deep water is shown by using a variational formulation.

The study of the regularity of water waves is an interesting problem, which may serve as a rigorous justification when approximating small-amplitude waves by their truncated power series expansions. We refer to [7, 20, 29] where first-order approximations of the wave profile are considered when studying the paths of the water particles located beneath a periodic wave traveling above an ocean of infinite depth. The results of [7, 20, 29] are improved in [6, 11, 19] in the case of irrotational waves by describing the exact geometry of the actual particle paths.

Concerning the regularity of gravity periodic water waves of finite depth it is first shown in [9] that the streamlines beneath the surface of traveling waves having a vorticity function with Hölder continuous first derivative are real analytic. Even more, if the vorticity function is real analytic the authors prove that the wave profile is also real analytic. It should be mentioned that one can weaken the assumption on the vorticity function, cf. 30, to prove analyticity of the streamlines underneath the wave profile for waves with merely bounded vorticity function. A similar result in this direction is obtained in [10], where a general theory for weak solutions of the water wave problem is developed. The regularity results for gravity water waves of finite depth are shown to still hold true when considering capillary-gravity [22, 24] and purely capillary periodic waves [4, 23].

The goal of this paper is to prove similar results for irrotational deep-water waves without stagnation points in both the solitary and periodic cases. In contrast to the finite depth case, when the main difficulties are caused by the regularity of the vorticity function [9, 10, 30, or additionally by the nonlinearities due to surface tension [4, 22, 23, in the present paper we have to deal with an unbounded geometry. As a consequence we lose compact embedding properties since Arzelà-Ascoli fails. Moreover, solution operators corresponding to elliptic boundary value problems are generally no longer Fredholm, cf. 28, a situation which does not occur in the finite-depth case.

Our first main result, Theorem 2.1, states that a deep-water wave with Hölder continuous vorticity function has smooth profile and all streamlines underneath the surface are smooth. Moreover, if the vorticity is analytic, then so is the wave profile too. The first claim is proved by using a finite difference approach as in 10 to eliminate the vorticity function from the problem, while in the case of a real analytic vorticity function we employ a theorem from [25. In order to prove our second main result, Theorem 4.1 , we exploit the invariance of the problem with respect to translations in the horizontal coordinate. This property makes it possible for us to introduce an additional parameter into the problem, in a similar manner as in [3, 9, 15, 22, 23, 30], and show, for periodic waves having a vorticity function which is integrable against a cubic weight, that the streamlines beneath the wave profile are real analytic. We shall prove this theorem by reducing the problem to the study of the invertibility of the solution operator to a certain elliptic Dirichlet problem, which suits the choice of weighted Sobolev spaces. 
2. The governing equations. Let $(x, y, z)$ denote the standard Cartesian coordinates and consider water waves propagating in the $x$-direction such that the motion of the fluid is identical in the $z$-direction. The coordinate $y$ is used to indicate the height of the particles within the wave. With this assumption, the water wave problem reduces to the study of a two-dimensional flow in the $(x, y)$-plane which is located in the region bounded by above by the wave profile $y=\eta(t, x)$. To analyse at the same time solitary and periodic gravity water waves we set $\mathbb{X}$ to be either $\mathbb{R}$ or $\mathbb{S}$, where $\mathbb{S}$ is the unit circle, and we identify functions on $\mathbb{S}$ with $2 \pi$-periodic functions on $\mathbb{R}$.

We present now the equations governing our problem. By mass conservation we have

$$
u_{x}+v_{y}=0 \text { in } \Omega_{\eta},
$$

where $(u, v)$ stands for the velocity field within the fluid domain

$$
\Omega_{\eta}:=\{(x, y): x \in \mathbb{X} \text { and }-\infty<y<\eta(x)\} .
$$

Furthermore, the influence of viscosity is neglected, so that the motion of the fluid can be described by Euler's equations:

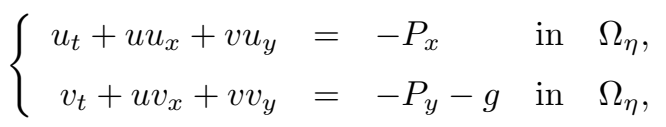

where we use $P$ to denote the dynamic pressure and $g$ for the gravity constant. A justification for considering water as an inviscid flow is presented in 27. At the wave surface we impose two kinematic boundary conditions:

$$
\begin{gathered}
P=P_{0} \quad \text { on } \quad y=\eta(t, x), \\
v=\eta_{t}+u \eta_{x} \quad \text { on } \quad y=\eta(t, x) .
\end{gathered}
$$

Neglecting surface tension, condition (2.1c) is obtained by setting the pressure on $y=$ $\eta(t, x)$ to be equal to the atmospheric pressure $P_{0}$, and the second condition (2.1d) relies on the assumption that particles located at the free boundary remain there for all times. For periodic waves we impose the far-field condition

$$
(u, v) \rightarrow(0,0) \quad \text { as } y \rightarrow-\infty \text { uniformly in } x,
$$

meaning that there is no flow at great depths. On the other hand, for solitary water waves we replace (2.1e) by two conditions, since we must also prescribe the behavior of $\eta$ at $\pm \infty$ :

$$
\left\{\begin{array}{l}
(u, v) \rightarrow(0,0) \text { as }|(x, y)| \rightarrow \infty, \\
\eta \rightarrow 0 \text { as }|x| \rightarrow \infty
\end{array}\right.
$$

Since we are interested in traveling wave solutions of problem (2.1), we presuppose that there exists a positive real number $c$, the speed of wave propagation, such that $(\eta, u, v, P)$ depend on $(t, x, y)$ in the following way:

$$
\eta(t, x)=\eta(x-c t), \quad(u, v, P)(t, x, y)=(u, v, P)(x-c t, y) .
$$


This hypothesis simplifies the problem (2.1) considerably and reduces it, in the case of periodic traveling waves, to the problem consisting of

$$
\left\{\begin{aligned}
(u-c) u_{x}+v u_{y} & =-P_{x} & & \text { in } \Omega_{\eta}, \\
(u-c) v_{x}+v v_{y} & =-P_{y}-g & & \text { in } \Omega_{\eta}, \\
u_{x}+v_{y} & =0 & & \text { in } \Omega_{\eta}, \\
P & =P_{0} & & \text { on } y=\eta(x), \\
v & =(u-c) \eta^{\prime} & & \text { on } y=\eta(x),
\end{aligned}\right.
$$

and (2.1el) [resp. $\left(2.1 \mathrm{e}^{\prime}\right)$ for solitary traveling waves].

In the remainder of this paper we consider classical solutions $(u, v, P, \eta)$ of (2.2) and (2.1e) [resp. $\left.\left(2.1 \mathrm{e}^{\prime}\right)\right]$ with the following regularity:

$$
(u, v, P, \eta) \in C^{1+\alpha}\left(\bar{\Omega}_{\eta}\right) \times C^{1+\alpha}\left(\bar{\Omega}_{\eta}\right) \times C^{1+\alpha}\left(\bar{\Omega}_{\eta}\right) \times C^{2+\alpha}(\mathbb{X}),
$$

for some constant $\alpha \in(0,1)$. The existence of water wave solutions in this class has been established in 31 for the periodic case. Finally, we presuppose that the wave has no stagnation points, that is,

$$
u-c<0 \quad \text { in } \bar{\Omega}_{\eta} .
$$

Making use of the incompressibility condition and (2.4) we introduce the stream function $\psi$ by the relation

$$
\psi(x, y):=-\int_{y}^{\eta(x)}(u(x, s)-c) d s, \quad(x, y) \in \bar{\Omega}_{\eta} .
$$

The function $\psi$ is twice differentiable, with derivatives $\psi_{x}, \psi_{y} \in C^{1+\alpha}\left(\bar{\Omega}_{\eta}\right)$. In fact $\psi \equiv 0$ on the wave surface $y=\eta(x), \psi_{y}=u-c<0$, and $\psi_{x}=-v$ in $\bar{\Omega}_{\eta}$. The last relation is obtained by differentiating into the integral and using the mass conservation (2.1a):

$$
\begin{aligned}
\psi_{x}(x, y) & =-\int_{y}^{\eta(x)} u_{x}(x, s) d s-(u(x, \eta(x))-c) \eta^{\prime}(x)=\int_{y}^{\eta(x)} v_{y}(x, s) d s-v(x, \eta(x)) \\
& =v(x, \eta(x))-v(x, y)-v(x, \eta(x))=-v(x, y) .
\end{aligned}
$$

A simple consequence of (2.4) is the fact that the streamlines, i.e. the level curves of $\psi$, are $C^{2+\alpha}$-graphs over $\mathbb{X}$. This may be easily seen by using the implicit function theorem together with (2.4). We show in our first main result, Theorem 2.1] that even more all streamlines are smooth. To prove this result we introduce the vorticity $\omega \in C^{\alpha}\left(\bar{\Omega}_{\eta}\right)$ by the relation

$$
\omega:=u_{y}-v_{x} .
$$

We show now that there exists a function $\gamma \in C^{\alpha}((-\infty, 0])$, called the vorticity function, which makes it possible to express the vorticity in dependence of the stream function, by the relation $\omega(x, y)=\gamma(-\psi(x, y))$ for all $(x, y) \in \bar{\Omega}_{\eta}$. In order to find $\gamma$, we transform the wave domain on the half-plane $\Omega:=\mathbb{X} \times(-\infty, 0)$ by using the hodograph transformation $\Phi: \bar{\Omega}_{\eta} \rightarrow \bar{\Omega}$ defined by

$$
\Phi(x, y):=(q, p)(x, y):=(x,-\psi(x, y)), \quad(x, y) \in \bar{\Omega}_{\eta} .
$$


Condition (2.4) guarantees that $\Phi$ is indeed a diffeomorphism of class $C^{2+\alpha}$. We proceed now and identify the vorticity function. Denote by $\bar{\omega}$ the vorticity in the new coordinates, that is, $\bar{\omega}:=\omega \circ \Phi^{-1}$. We make a change of variables and get, due to $\psi_{y}(x, y)=-\operatorname{det} \partial \Phi(x, y)>0$, that the distributional derivative of $\bar{\omega}$ with respect to $q$ satisfies

$$
\left\langle\partial_{q} \bar{\omega} \mid \bar{\phi}\right\rangle=-\int_{\Omega} \bar{\omega} \partial_{q} \bar{\phi} d(q, p)=\int_{\Omega_{\eta}} \omega \psi_{y} \partial_{q} \bar{\phi} \circ \Phi d(x, y)=\int_{\Omega_{\eta}} \omega(u-c) \partial_{q} \bar{\phi} \circ \Phi d(x, y)
$$

for all test functions $\bar{\phi} \in C_{0}^{\infty}(\Omega)$. Note that the mapping $\phi:=\bar{\phi} \circ \Phi$ is no longer smooth, but it still has compact support. Taking into consideration that the Jacobian of $\Phi$ is given by

$$
\partial \Phi(x, y)=\frac{\partial(q, p)}{\partial(x, y)}=\left[\begin{array}{cc}
1 & 0 \\
v & c-u
\end{array}\right],
$$

we obtain that $\partial_{x} \phi=\partial_{q} \bar{\phi} \circ \Phi+v \partial_{p} \bar{\phi} \circ \Phi, \partial_{y} \phi=(c-u) \partial_{p} \bar{\phi} \circ \Phi$, and therefore we conclude that $\psi_{y} \partial_{q} \bar{\phi} \circ \Phi=(u-c) \partial_{x} \phi+v \partial_{y} \phi$. Invoking Euler's equations and the definition of the vorticity (2.5), integration by parts yields

$$
\begin{aligned}
\left\langle\partial_{q} \bar{\omega} \mid \bar{\phi}\right\rangle & =\int_{\Omega_{\eta}}\left(u_{y}-v_{x}\right)\left((u-c) \partial_{x} \phi+v \partial_{y} \phi\right) d(x, y) \\
& =\int_{\Omega_{\eta}}\left((u-c) u_{x}+v u_{y}\right) \partial_{y} \phi d(x, y)-\int_{\Omega_{\eta}}\left((u-c) v_{x}+v v_{y}\right) \partial_{x} \phi d(x, y) \\
& =\int_{\Omega_{\eta}}(P+g y)_{y} \partial_{x} \phi d(x, y)-\int_{\Omega_{\eta}} P_{x} \partial_{y} \phi d(x, y) \\
& =\int_{\Omega_{\eta}} P_{x} \partial_{y} \phi d(x, y)-\int_{\Omega_{\eta}} P_{x} \partial_{y} \phi d(x, y)=0 .
\end{aligned}
$$

In order to define the vorticity function, we choose a test function $\phi_{1} \in C_{0}^{\infty}(\mathbb{X})$ having integral mean equal to 1 . Given $\bar{\phi} \in C_{0}^{\infty}(\Omega)$, the function

$$
\bar{\phi}_{2}(q, p):=\bar{\phi}(q, p)-\phi_{1}(q) \int_{\mathbb{X}} \bar{\phi}(s, p) d s
$$

is again a test function $C_{0}^{\infty}(\Omega)$, and moreover, $\bar{\phi}_{2}(\cdot, p)$ has zero integral mean for all $p \leq 0$. Hence, the map $\bar{\phi}_{3}: \Omega \rightarrow \mathbb{R}$, defined by

$$
\bar{\phi}_{3}(q, p)=\int_{q_{0}}^{q} \bar{\phi}_{2}(s, p) d s,
$$

with $q_{0}$ chosen such that it does not belong to the projection of the support of $\phi_{2}$ on $\mathbb{R}$ if $\mathbb{X}=\mathbb{R}\left[\right.$ resp. $q_{0}=0$ if $\left.\mathbb{X}=\mathbb{S}\right]$ belongs to $C_{0}^{\infty}(\Omega)$ and $\partial_{q} \bar{\phi}_{3}=\bar{\phi}_{2}$. According to $\partial_{q} \bar{\omega}=0$, we have $\left\langle\bar{\omega} \mid \bar{\phi}_{2}\right\rangle=\left\langle\bar{w} \mid \partial_{q} \bar{\phi}_{3}\right\rangle=-\left\langle\partial_{q} \bar{w} \mid \bar{\phi}_{3}\right\rangle=0$, and so

$$
\begin{aligned}
\langle\bar{w} \mid \bar{\phi}\rangle & =\int_{\Omega} \bar{w}(q, p) \phi_{1}(q) \int_{\mathbb{X}} \bar{\phi}(s, p) d s d q d p=\int_{-\infty}^{0} \int_{\mathbb{X}}\left(\int_{\mathbb{X}} \bar{w}(q, p) \phi_{1}(q) d q\right) \bar{\phi}(s, p) d s d p \\
& =\int_{\Omega} \gamma(p) \bar{\phi}(q, p) d(q, p)=\langle\gamma \mid \bar{\phi}\rangle,
\end{aligned}
$$


where $\gamma$ is the function

$$
\gamma(p):=\int_{\mathbb{X}} \bar{w}(q, p) \phi_{1}(q) d q, \quad-\infty<p \leq 0 .
$$

We have thus shown that $\bar{\omega}=\gamma$. In view of $\bar{\omega} \in C^{\alpha}(\bar{\Omega})$ and $\phi_{1} \in C_{0}^{\infty}(\mathbb{X})$ we conclude that the vorticity function $\gamma$ is a Hölder continuous map, that is, $\gamma \in C^{\alpha}((-\infty, 0])$. Returning to the physical coordinates, we have shown that

$$
\Delta \psi(x, y)=\omega(x, y)=\bar{w}(x,-\psi(x, y))=\gamma(-\psi(x, y))
$$

for all $(x, y) \in \Omega_{\eta}$. Furthermore, by Bernoulli's principle, the quantity

$$
\frac{(u-c)^{2}+v^{2}}{2}+g y+P-\int_{0}^{\psi} \gamma(-s) d s
$$

is constant in $\Omega_{\eta}$, so that on $y=\eta(x)$ we get $|\nabla \psi|^{2}+2 g y=Q$, with some constant $Q$. Summarising, we found out that the function $\psi$ solves the following problem:

$$
\left\{\begin{aligned}
\Delta \psi & =\gamma(-\psi) & & \text { in } \Omega_{\eta}, \\
|\nabla \psi|^{2}+2 g y & =Q & & \text { on } y=\eta(x), \\
\psi & =0 & & \text { on } y=\eta(x),
\end{aligned}\right.
$$

which is equivalent to system (2.2) The far-field condition (2.1e) may also be expressed in terms of $\psi$ :

$$
\nabla \psi \rightarrow(0,-c) \text { as } y \rightarrow-\infty \text { uniformly in } x,
$$

while $\left(2.1 \mathrm{e}^{\prime}\right)$ can be rewritten as

$$
\left\{\begin{array}{l}
\nabla \psi \rightarrow(0,-c) \quad \text { as }|(x, y)| \rightarrow \infty, \\
\eta \rightarrow 0 \quad \text { as }|x| \rightarrow \infty
\end{array}\right.
$$

The first main result of this paper is the following theorem, saying that both solitary and periodic gravity water waves traveling above an ocean of infinite depth and having a uniformly Hölder continuous vorticity function are smooth and have smooth streamlines.

Theorem 2.1. Let $(u, v, P, \eta)$ be a solution of (2.2) and (2.1e) [resp. (2.2) and $\left(2.1 \mathrm{e}^{\prime}\right)$ ] satisfying (2.3), (2.4), and having a uniformly Hölder continuous vorticity function $\gamma$. Then, the wave profile $\eta$ and all streamlines beneath the wave surface are smooth. Moreover, if $\gamma$ is real analytic, then the wave profile $\eta$ is also real analytic.

Proof. Assume that $\gamma$ is a real analytic function. In order to apply the regularity results for free boundaries arising in elliptic problems, as presented in [25], we define $\Omega_{-}:=\{(x, y): y<\eta(x)\}, \Omega_{+}:=\{(x, y): y>\eta(x)\}, \psi_{+}=0$, and $\psi_{-}=\psi$. Then, we have

$$
\left\{\begin{array}{lll}
\Delta \psi_{-}-\gamma(-\psi)=0 & \text { in } & \Omega_{-}, \\
\Delta \psi_{+}=0 & \text { in } & \Omega_{+}, \\
\psi_{+}=\psi_{-}=0 & \text { on } & y=\eta(x) .
\end{array}\right.
$$

Furthermore, the second and third boundary condition of (2.6) imply, cf. [9], that

$$
f\left((x, y), \partial_{\nu} \psi_{-}, \partial_{\nu} \psi_{+}\right):=\left(\partial_{\nu} \psi_{-}\right)^{2}+2 g y-Q=0 \quad \text { on } \quad y=\eta(x),
$$


with $\nu$ the outward unit normal at $\partial \Omega_{-}$. Since $\left|\partial_{\nu} \psi_{-}\right|^{2}=\left|\nabla \psi_{-}\right|^{2}$ on $y=\eta(x)$, we infer from (2.4) that $\left|\partial_{\nu} \psi_{-}\right|^{2}>0$. Consequently, we may apply Theorem 3.2 in [25] and the remark following it, and obtain that $\eta$ is a real analytic function.

The smoothness of the streamlines and of $\eta$ when $\gamma$ is only Hölder continuous is a direct consequence of Proposition 3.1 below.

3. Proof of Theorem 2.1. It is quite intricate to prove the remaining part of the assertion of Theorem 2.1 by using only the new formulation (2.6) since the vorticity function is merely Hölder continuous and the regularity of the domain $\Omega_{\eta}$ depends on $\eta$. That is why we reformulate our problem by using the hodograph coordinates $\Phi$. To do so, we let $h: \bar{\Omega} \rightarrow \mathbb{R}$ be the function with $h(q, p):=y$ for $(q, p) \in \bar{\Omega}$. In hodograph coordinates, (2.6) is a nonlinear boundary value problem for a quasilinear elliptic equation with $h$ as unknown,

$$
\left\{\begin{aligned}
\frac{1+h_{q}^{2}}{h_{p}^{3}} h_{p p}-2 \frac{h_{q}}{h_{p}^{2}} h_{p q}+\frac{1}{h_{p}} h_{q q}-\gamma(p) & =0 \quad \text { in } \quad \Omega, \\
\frac{1+h_{q}^{2}}{h_{p}^{2}}+2 g h & =Q \quad \text { on } \quad p=0,
\end{aligned}\right.
$$

and for $\mathbb{X}=\mathbb{S}$,

$$
\nabla h \rightarrow(0,1 / c) \text { as } p \rightarrow-\infty \text { uniformly in } q,
$$

while for $\mathbb{X}=\mathbb{R}$ we reformulate $\left(2.1 \mathrm{e}^{\prime}\right)$ as

$$
\left\{\begin{array}{l}
\nabla h \rightarrow(0,1 / c) \quad \text { as }|(q, p)| \rightarrow \infty, \\
h(q, 0) \rightarrow 0 \quad \text { as }|q| \rightarrow \infty .
\end{array}\right.
$$

Additionally, we can use $h$ to define a parametrization of the streamlines of the gravity wave, since by the definition of $h$, we have that $y=h(x,-\psi(x, y))$ for all $(x, y) \in \bar{\Omega}_{\eta}$, whence, the streamline $\psi^{-1}(\{-p\})$, with $p \leq 0$, is parametrized by the map $q \mapsto h(q, p)$. Since $\psi^{-1}(\{0\})$ is exactly the wave profile, which is the graph of $\eta$, we have that $\eta(q)=$ $h(q, 0)$, which explains the boundary behavior of $h(q, 0)$ for $|q| \rightarrow \infty$, when considering solitary waves. Note that though $h$ is unbounded, it appears in the first partial differential equation (3.1) only through its derivatives, which satisfy

$$
h_{q}=-\frac{v}{c-u} \circ \Phi^{-1} \in C^{1+\alpha}(\bar{\Omega}) \quad \text { and } \quad h_{p}=\frac{1}{c-u} \circ \Phi^{-1} \in C^{1+\alpha}(\bar{\Omega}) .
$$

Furthermore, the vorticity function depends only upon the coordinate $p$, and

$$
\inf _{\Omega} h_{p}(q, p)>0 .
$$

Thus, proving Theorem 2.1 reduces to showing that $q \mapsto h(q, p)$ is smooth for all $p \leq 0$. We will prove instead the stronger result, Proposition 3.1 which does not take into account some of the far-field boundary conditions satisfied by $h$. We shall state the result for $\mathbb{X}=\mathbb{R}$, the situation when $\mathbb{X}=\mathbb{S}$ being a particular case of Proposition 3.1, whence, let $\Omega=\mathbb{R} \times(-\infty, 0)$ in the remainder of this section.

Proposition 3.1. Let $h: \bar{\Omega} \rightarrow \mathbb{R}$ be a solution of (3.1) with partial derivatives satisfying (3.3) and (3.4). Then, the mapping $q \mapsto h(q, p)$ is smooth for all $p \leq 0$. 
Proof. We shall prove by using the induction principle that for all $n \geq 0$,

$$
\frac{\partial^{\left(\lambda_{1}, \lambda_{2}\right)} h}{\partial q^{\lambda_{1}} \partial p^{\lambda_{2}}} \in C^{\alpha}(\bar{\Omega}) \text { for all } \lambda_{1}+\lambda_{2}=n+2 \text { with } 0 \leq \lambda_{2} \leq 2 .
$$

The assertion when $n=0$ follows directly from (3.3). In order to finish the proof, presuppose that (3.5) is true for all $0 \leq k \leq n$ and prove it for $n+1$. Using the induction assumption, it is not difficult to see that it suffices to show that $\partial^{n+1} h / \partial q^{n+1} \in C^{2+\alpha}(\bar{\Omega})$. To this end we define the rational functions $a, b: \mathbb{R} \times(0, \infty) \rightarrow \mathbb{R}, c:(0, \infty) \rightarrow \mathbb{R}$, and $d: \mathbb{R} \rightarrow \mathbb{R}$ by the formula

$$
a(x, y)=\frac{1+x^{2}}{y^{3}}, \quad b(x, y)=\frac{x}{y^{2}}, \quad c(y)=\frac{1}{y}, \quad d(x)=1+x^{2}, \quad(x, y) \in \mathbb{R} \times(0, \infty) .
$$

With this notation, $h$ solves the following system:

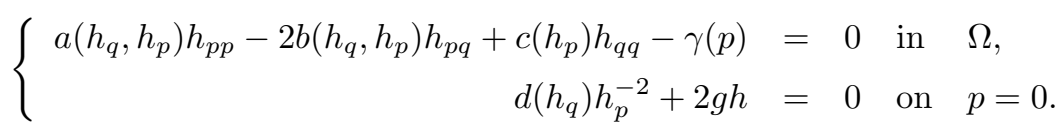

The conditions (3.3) and (3.4) ensure that the first equation of (3.6) is uniformly elliptic. The induction assumption allows us to differentiate the system (3.6) $n$ times with respect to $q$ and find that $\widetilde{h}:=\partial^{n} h / \partial q^{n}$ solves, if $n \geq 1$, the following problem:

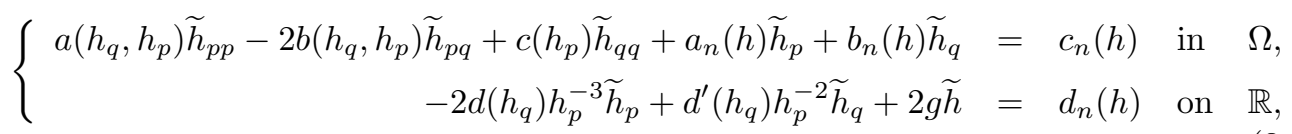

where $a_{n}, d_{n}, c_{n}$ are rational functions depending only on the derivatives $\partial^{\left(\lambda_{1}, \lambda_{2}\right)} h$ with $1 \leq \lambda_{1}+\lambda_{2} \leq n+1$ and $0 \leq \lambda_{2} \leq 2$, while $d_{n}$ is also rational, but depends on the derivatives $\partial^{\left(\lambda_{1}, \lambda_{2}\right)} h$ with $1 \leq \lambda_{1}+\lambda_{2} \leq n$ and $0 \leq \lambda_{2} \leq 1$. Furthermore, if we multiply $a_{n}, b_{n}, c_{n}$, and $d_{n}$ by $h_{p}^{n+3}$ the products are polynomials in the above-mentioned variables. If $n=0$, then $\widetilde{h}=h$ and $h$ solves (3.6).

Then, for each $l \in \mathbb{N}$, we consider the functions $h_{l}, \widetilde{h}_{l}, w_{l}: \bar{\Omega} \rightarrow \mathbb{R}$, given by $h_{l}(q, p):=$ $h\left(q+l^{-1}, p\right), \widetilde{h}_{l}:=\partial^{n} h_{l} / \partial q^{n}$, and

$$
w_{l}(q, p):=\frac{\widetilde{h}_{l}(q, p)-\widetilde{h}(q, p)}{l^{-1}}, \quad(q, p) \in \bar{\Omega} .
$$

We infer from

$$
\left|w_{l}(q, p)\right|=\frac{\left|\widetilde{h}\left(q+l^{-1}, p\right)-\widetilde{h}(q, p)\right|}{l^{-1}} \leq\left\|\widetilde{h}_{q}\right\|_{C(\bar{\Omega})}
$$

that $w_{l} \in C^{2+\alpha}(\bar{\Omega})$ (this estimate is necessary only for $n=0$, because when $n \geq 1$ we know from the induction assumption that $\widetilde{h} \in C^{2+\alpha}(\bar{\Omega})$ ).

We prove the assertion only when $n \geq 1$ (the case $n=0$ follows in a similar manner). Making use of the invariance of (3.6) with respect to translations in the $q$ variable, we subtract from both equations of (3.7) the same relations but evaluated at $\left(q+l^{-1}, p\right)$. If we divide the new identities by $l^{-1}$ we observe that $w_{l}$ solves the elliptic boundary value 
problem

$$
\left\{\begin{array}{l}
a\left(h_{q}, h_{p}\right) w_{l, p p}-2 b\left(h_{q}, h_{p}\right) w_{l, p q}+c\left(h_{p}\right) w_{l, q q}+a_{n}(h) w_{l, p}+b_{n}(h) w_{l, q}=-A_{l}(h) \text { in } \Omega, \\
-2 d\left(h_{q}\right) h_{p}^{-3} w_{l, p}+d^{\prime}\left(h_{q}\right) h_{p}^{-2} w_{l, q}+2 g w_{l}=-B_{l}(h) \quad \text { on } p=0,
\end{array}\right.
$$

where $A_{l} \in C^{\alpha}(\bar{\Omega})$ and $B_{l} \in C^{1+\alpha}(\bar{\Omega})$ are given respectively by

$$
\begin{gathered}
A_{l}(h):=\frac{a\left(h_{q}, h_{p}\right)-a\left(h_{l, q}, h_{l, p}\right)}{l^{-1}} \widetilde{h}_{l, p p}-2 \frac{b\left(h_{q}, h_{p}\right)-b\left(h_{l, q}, h_{l, p}\right)}{l^{-1}} \widetilde{h}_{l, p q}+\frac{c_{n}\left(h_{l}\right)-c_{n}(h)}{l^{-1}} \\
+\frac{c\left(h_{q}, h_{p}\right)-c\left(h_{l, q}, h_{l, p}\right)}{l^{-1}} \widetilde{h}_{l, q q}+\frac{a_{n}(h)-a_{n}\left(h_{l}\right)}{l^{-1}} \widetilde{h}_{l, p}+\frac{b_{n}(h)-b_{n}\left(h_{l}\right)}{l^{-1}} \widetilde{h}_{l, q}, \\
B_{l}(h):=\frac{2 d\left(h_{l, q}\right) h_{l, p}^{-3}-2 d\left(h_{q}\right) h_{p}^{-3}}{l^{-1}} \widetilde{h}_{l, p}+\frac{d^{\prime}\left(h_{q}\right) h_{p}^{-2}-d^{\prime}\left(h_{l, q}\right) h_{l, p}^{-2}}{l^{-1}}+\frac{d_{n}\left(h_{l}\right)-d_{n}(h)}{l^{-1}} .
\end{gathered}
$$

Clearly, $\widetilde{h}_{l}$ is uniformly bounded in $C^{2+\alpha}(\bar{\Omega})$ independently of $l$. Since all the partial derivatives $\partial^{\left(\lambda_{1}, \lambda_{2}\right)} h$, with $1 \leq \lambda_{1}+\lambda_{2} \leq n+1$ and $0 \leq \lambda_{2} \leq 2$, are bounded in $C^{\alpha}(\bar{\Omega})$ and $a_{n}, b_{n}, c_{n}$ are rational functions, we apply the mean value theorem as in [14, Lemma 1.2], and get that $A_{l}$ is uniformly bounded in $C^{\alpha}(\bar{\Omega})$ by a constant independent of $l$. Similarly, $B_{l}$ is uniformly bounded in $C^{1+\alpha}(\bar{\Omega})$. Moreover, in view of (3.4), we see that the coefficient of $w_{l, p}$ in the second equation of (3.9) is bounded away from zero, and, by the induction assumption, we have, cf. 11, Theorem 7.3], the following Schauder estimates for $w_{l}$ :

$$
\left\|w_{l}\right\|_{C^{2+\alpha}(\bar{\Omega})} \leq C\left(\left\|A_{l}\right\|_{C^{\alpha}(\bar{\Omega})}+\left\|B_{l}\right\|_{C^{1+\alpha}(\bar{\Omega})}+\left\|w_{l}\right\|_{C(\bar{\Omega})}\right),
$$

with some constant independent of $l \in \mathbb{N}$. Invoking (3.8), we have found a uniform bound for the $C^{2+\alpha}$-norm of $w_{l}$. Since $w_{l}$ converges to $\partial_{q} \widetilde{h}$ pointwise, this shows that $\partial^{n+1} h / \partial q^{n+1} \in C^{2+\alpha}(\bar{\Omega})$, and the proof is completed.

4. Analyticity of the streamlines for periodic traveling waves with Hölder continuous vorticity function. In this last section we prove our second main result, Theorem 4.1] Therefore, we set $\Omega:=\mathbb{S} \times(-\infty, 0)$ and define the weight $\rho(q, p):=(1+$ $\left.p^{2}\right)^{1 / 2},(q, p) \in \Omega$, which measures the distance from the boundary $p=0$. Furthermore, we fix $r>2$ and, following [28], we define for all $l \in \mathbb{N}$ and $\beta \in \mathbb{R}$, the weighted Sobolev spaces

$$
H_{l, \beta}^{r}(\Omega):=\left\{u \in \mathcal{D}^{\prime}(\Omega): \rho^{\beta+|\lambda|} D^{\lambda} u \in L^{r}(\Omega) \text { for all }|\lambda| \leq l\right\},
$$

which is a Banach space with the norm

$$
\|u\|_{H_{l, \beta}^{r}(\Omega)}:=\sum_{|\lambda| \leq l}\left\|\rho^{|\lambda|+1} D^{\lambda} u\right\|_{L^{r}(\Omega)} .
$$

Of particular interest for us are the spaces $X:=H_{2,1}^{r}(\Omega)$ and $Y:=H_{0,3}^{r}(\Omega)$. In the notation used in [2], these spaces are exactly $H_{2,1}^{r}(\Omega)=W_{3}^{2, r}(\Omega)$ and $H_{0,3}^{r}(\Omega)=W_{3}^{0, r}(\Omega)$. Note that the functions belonging to $X$ and their derivatives are $L^{r}$-integrable on compact sets, which means that the right trace space $Z$ is

$$
Z:=B_{r r}^{2-1 / r}(\mathbb{S})
$$


whereby $B_{r r}^{2-1 / r}(\mathbb{S})$ denotes the usual Besov space; cf. 32]. The main result of this section is the following theorem.

TheOREm 4.1. Let $\alpha>1 / 2$ and pick a solution $(u, v, P, \eta)$ of (2.2) and (2.1e) which has a Hölder continuous vorticity function $\gamma \in C^{\alpha}((-\infty, 0])$. We denote by $h^{0}$ the corresponding solution of (3.1) and (3.2a). Additionally, we presuppose that:

(i) $|p|^{3} \gamma \in L^{r}((-\infty, 0))$.

(ii) Letting $H(p):=h^{0}(0, p)$ for $p \leq 0$, we assume that $h^{0}-H \in X$.

(iii) Uniformly in $q$ :

$$
|p|\left[h_{q}^{0} h_{p p}^{0}-h_{p}^{0} h_{p q}^{0}\right] \rightarrow_{p \rightarrow-\infty} 0 \quad \text { and } \quad|p|\left[2 h_{p}^{0} h_{q q}^{0}+3 \gamma(p)\left(h_{p}^{0}\right)^{2}-2 h_{q}^{0} h_{p q}^{0}\right] \rightarrow_{p \rightarrow-\infty} 0 .
$$

Then each streamline beneath the surface is real analytic.

REMARK 4.2. The condition $(i)$ of the theorem ensures that $\gamma \in H_{0,3}^{r}(\Omega)$. This property is never satisfied when we consider solitary traveling wave solutions of (2.2), that is, when $\Omega=\mathbb{R} \times(-\infty, 0)$. This constitutes an impediment when trying to apply the methods presented below in that case. In fact, a similar condition was imposed in [31] to ensure existence of periodic gravity deep-water traveling waves. Moreover, the second condition (ii) is satisfied by symmetric waves with negative and nonincreasing vorticity; cf. [31, Lemma 2.1] and [8].

Since the streamlines are parametrized by $h(\cdot, p), p<0$, it suffices to show that the mapping $a \mapsto \tau_{a} h^{0}(q, p),(q, p) \in \Omega$, with $\tau_{a} h^{0}$ the translation introduced in [9, that is,

$$
\tau_{a} h^{0}(p, q):=h^{0}(q+a p, p) \quad \text { for }(q, p) \in \Omega \text { and } a \in \mathbb{R},
$$

is real analytic in a small neighborhood of 0 in $\mathbb{R}$. The difficulty in our case is due to the unboundedness of $\Omega$, since solution operators to Dirichlet boundary value problems are generally not Fredholm; cf. 28. This is the reason why we chose to work within the weighted Sobolev spaces setting.

Proof of Theorem 4.1. We begin by defining the operator $F=\left(F_{1}, F_{2}\right): X \rightarrow Y \times Z$ by setting

$$
\begin{aligned}
& F_{1}(u):=\left(1+u_{q}^{2}\right)\left(u_{p p}+H^{\prime \prime}\right)-2 u_{q}\left(u_{p}+H^{\prime}\right) u_{p q}+\left(u_{p}+H^{\prime}\right)^{2} u_{q q}-\left(u_{p}+H^{\prime}\right)^{3} \gamma(p), \\
& F_{2}(u):=\frac{1+h_{0, q}^{2}}{2 g h_{0, p}^{2}}+u+H(0)-Q / 2 g,
\end{aligned}
$$

for $u \in X$, where $H^{\prime}$ and $H^{\prime \prime}$ are the first and second order derivatives of $H$, respectively. Note that with our choice of $\alpha$ we may pick $r>2$ such that $1+\alpha>2-1 / r$, meaning that $C^{1+\alpha}(\mathbb{S}) \subset Z$. By the definition of $F$, the function $u_{0}:=h_{0}-H \in X$ is a solution of $F(u)=0$, whence, the condition $(i)$ imposed on $\gamma$ together with the Sobolev embedding $W_{3}^{2, r}(\Omega) \subset W^{2, r}(\Omega) \subset C^{1}(\bar{\Omega})$ ensure that the mapping $F$ is well-defined. Denote by $K: \mathbb{R} \times X \rightarrow Y$ the operator satisfying $K\left(a, \tau_{a} u\right):=F_{1}\left(\tau_{a} u\right)-\tau_{a} F_{1}(u)$ for all $(a, u) \in \mathbb{R} \times X$. Given $(a, u) \in \mathbb{R} \times X$, we have the following formula:

$$
K(a, u):=2 a u_{q p}-a^{2} u_{q q}+\gamma(p)\left[3 a u_{q}\left(u_{p}+H^{\prime}\right)^{2}-3 a^{2} u_{q}^{2}\left(u_{p}+H^{\prime}\right)+a^{3} u_{q}^{3}\right] .
$$

Finally, let $\Psi: \mathbb{R} \times X \rightarrow Y \times Z$ denote the mapping given by

$$
\Psi(a, u):=F(u)-(K(a, u), 0) .
$$


By definition of $\Psi$ and of the translation $\tau_{a}$, we easily see that the curve $\left\{\left(a, \tau_{a} u^{0}\right): a \in\right.$ $\mathbb{R}\}$ consists entirely of solutions of the operator equation

$$
\Psi(a, u)=0 .
$$

If $\partial_{u} \Psi\left(0, u^{0}\right)$ is an isomorphism, that is, $\partial_{u} \Psi\left(0, u^{0}\right) \in \operatorname{Isom}(X, Y \times Z)$, then we may apply the implicit function theorem to conclude that the curve $\left\{\left(a, \tau_{a} u^{0}\right): a \in \mathbb{R}\right\}$ is real analytic in a neighborhood of $a=0$, which implies the desired assertion.

Setting $\partial_{u} \Psi\left(0, u^{0}\right)=:(L, T)$, we have

$$
\begin{aligned}
L u:= & u_{p p}+c^{-2} u_{q q}+\left(h_{q}^{0}\right)^{2} u_{p p}-2 h_{p}^{0} h_{q}^{0} u_{p q}+\left[\left(h_{p}^{0}\right)^{2}-c^{-2}\right] u_{q q} \\
& +2\left[h_{q}^{0} h_{p p}^{0}-h_{p}^{0} h_{p q}^{0}\right] u_{q}+\left[2 h_{p}^{0} h_{q q}^{0}+3 \gamma(p)\left(h_{p}^{0}\right)^{2}-2 h_{q}^{0} h_{p q}^{0}\right] u_{p}, \\
T u:= & \left.u\right|_{p=0}
\end{aligned}
$$

for $u \in X$. If we write $L=\partial_{p}^{2}+c^{-2} \partial_{q}^{2}+\sum_{|\alpha| \leq 2} a_{\alpha} \partial^{\alpha}$, then we infer from (3.2a) and the assumption (ii) of the theorem that

$$
\rho^{2-|\alpha|} a_{\alpha} \rightarrow_{p \rightarrow-\infty} 0 \quad \text { uniformly in } q .
$$

We may proceed now as in the proof of [28, Theorem 5.6], and obtain in our particular situation that the operator $(L, T)$ is Fredholm exactly when the limit operator

$$
\left(L_{\infty}, T_{\infty}\right):=\left(\partial_{p}^{2}+c^{-2} \partial_{q}^{2}, \operatorname{tr}\right),
$$

which is obtained by substituting each coefficient of $(L, T)$ by its limit as $p \rightarrow-\infty$, is Fredholm. Moreover, we have that the Fredholm index of these operators is the same. However, we infer from [2] that the limit operator is in fact an isomorphism, i.e., $\left(L_{\infty}, T_{\infty}\right) \in \operatorname{Isom}(X, Y \times Z)$. This shows that $\partial_{u} \Psi\left(0, u^{0}\right)$ is a Fredholm operator of index zero. Using the strong maximum principle, we show that $\partial_{u} \Psi\left(0, u_{0}\right)$ is injective. Indeed, if $(L u, T u)=0$ and $u \neq 0$, then $u$ cannot achieve a positive maximum in $\Omega$. Otherwise, we may regard $u \in W_{2}^{1}(\Omega)$ as a weak solution of the elliptic problem

$$
\partial_{i}\left(a_{i j} u_{j}\right)+a_{i} u_{i}=0
$$

where $\partial_{1}:=\partial_{q}, \partial_{2}:=\partial_{p}, a_{11}:=\left(h_{p}^{0}\right)^{2}, a_{12}:=-h_{p}^{0} h_{q}^{0}, a_{22}:=1+\left(h_{q}^{0}\right)^{2}$, and where

$$
\begin{aligned}
& a_{1}:=2\left[h_{q}^{0} h_{p p}^{0}-h_{p}^{0} h_{p q}^{0}\right]-\partial_{1} a_{11}-\partial_{2} a_{12}, \\
& a_{2}:=\left[2 h_{p}^{0} h_{q q}^{0}+3 \gamma(p)\left(h_{p}^{0}\right)^{2}-2 h_{q}^{0} h_{p q}^{0}\right]-\partial_{1} a_{12}-\partial_{2} a_{22} .
\end{aligned}
$$

The strong maximum principle for weak solutions [17, Theorem 8.19] implies that $u$ is constant, in contradiction to $u \in X$. On the other hand, if $u$ does not attain its supremum, there exists a sequence $\left(q_{n}, p_{n}\right)$ with $p_{n} \rightarrow-\infty$ and $\varepsilon>0$ such that $u\left(q_{n}, p_{n}\right)>\varepsilon$ for all $n \in \mathbb{N}$ ( $u \in X$ implies that $u$ is uniformly continuous). Hence, we may find $\delta>0$ such that still $u(p, q)>\varepsilon / 2$ if $(p, q) \in\left[q_{n}-\delta, q_{n}+\delta\right] \times\left[p_{n}-\delta, p_{n}+\delta\right]$, in contradiction with $u \in X$. Consequently, $\partial_{u} \Psi\left(0, u_{0}\right)$ is an isomorphism, and the desired assertion follows from the implicit function theorem.

Acknowledgement. The author would like to thank Joachim Escher for fruitful discussions which increased the quality of the paper. 


\section{REFERENCES}

[1] Agmon, S., Douglis, A., and L. Nirenberg. "Estimates near the boundary for solutions of elliptic partial differential equations satisfying general boundary conditions". I. Comm. Pure Appl. Math. 12, (1959): 623-727. MR0125307 (23:A2610)

[2] Amrouche, C. and S. Necăsová. "Laplace equation in the half-space with a nonhomogeneous Dirichlet boundary condition." Math. Bohem. 126, no. 2 (2001): 265-274. MR1844267 (2002e:35057)

[3] Angenent, S. "Parabolic equations for curves on surfaces." Ann. of Math. (2) 132, no. 2 (1990): 451-483. MR.1078266 (91k:35102)

[4] Craig, W. and A.-M. Matei. "On the regularity of the Neumann problem for the free surfaces with surface tension." Proc. Amer. Math. Soc. 135 (2007): 2497-2504. MR.2302570 (2008a:35297)

[5] Constantin, A. "On the deep water wave motion." J. Phys. A 34, no. 7 (2001): 1405-1417. MR 1819940 (2002b:76010)

[6] Constantin, A. "The trajectories of particles in Stokes waves." Invent. Math. 166, (2006): 523-535. MR 2257390(2007j:35240)

[7] Constantin, A., Ehrnström, M., and G. Villari. "Particle trajectories in linear deep-water waves." Nonlinear Anal. Real World Appl. 9, (2008): 1336-1344. MR2422547 (2009f:35265)

[8] Constantin, A. and J. Escher. "Symmetry of steady deep-water waves with vorticity." European J. Appl. Math. 15, (2004): 755-768. MR2144685 (2006b:76013)

[9] Constantin, A. and J. Escher. "Analyticity of periodic traveling free surface water waves with vorticity." Ann. of Math. (2) 173 (2011): 559-568. MR2753609

[10] Constantin, A. and W. Strauss. "Exact steady periodic water waves with vorticity." Comm. Pure Appl. Math. 57, no. 4 (2004): 481-527. MR.2027299 (2004i:76018)

[11] Constantin, A. and W. Strauss. "Pressure beneath a Stokes wave." Comm. Pure Appl. Math. 63, no. 4 (2010): 533-557. MR2604871

[12] Constantin, A. and W. Strauss. "Periodic traveling gravity water waves with discontinuous vorticity.", preprint.

[13] Dubreil-Jacotin, M.-L. "Sur la détermination rigoureuse des ondes permanentes périodiques d'ampleur finie." J. Math. Pures Appl. 13, (1934): 217-291.

[14] Escher, J. and B.-V. Matioc. "A moving boundary problem for periodic Stokesian Hele-Shaw flows." Interfaces Free Bound. 11, (2009): 119-137. MR2487025 (2010f:35448)

[15] Escher, J. and G. Simonett. "Analyticity of the interface in a free boundary problem." Math. Ann. 305, (1996): 439-459. MR.1397432 (98d:35242)

[16] Gerstner, F. "Theorie der Wellen samt einer daraus abgeleiteten Theorie der Deichprofile." Ann. Phys. 2, (1809): 412-445.

[17] Gilbarg, D. and T. S. Trudinger. Elliptic Partial Differential Equations of Second Order, New York: Springer-Verlag, 1998.

[18] Groves, M. D. and E. Wahlén. "On the existence and conditional energetic stability of solitary gravity-capillary surface waves on deep water." preprint.

[19] Henry, D. "The trajectories of particles in deep-water Stokes waves." Int. Math. Res. Not. Art. ID 23405 (2006): 1-13. MR2272104 (2007k:76017)

[20] Henry, D. "Particle trajectories in linear periodic capillary and capillary-gravity deep-water waves." J. Nonlinear Math. Phys. 14, no. 1 (2007): 1-7. MR2287829(2007k:76018)

[21] Henry, D. "On Gerstner's water wave." J. Nonlinear Math. Phys. 15, (2008): 87-95. MR2434727 (2009k:76020)

[22] Henry, D. "Analyticity of the streamlines for periodic travelling free surface capillary-gravity water waves with vorticity." SIAM J. Math. Anal. 42 (2010): 3103-3111. MR2763714

[23] Henry, D. "Regularity for steady periodic capillary water waves with vorticity.", preprint.

[24] Henry, D. "Analyticity of free surface for periodic traveling capillary-gravity water waves with vorticity.", preprint.

[25] Kinderlehrer, D., Nirenberg, L., and J. Spruck. "Regularity in elliptic free boundary value problems I." J. Anal. Math. 34, (1978): 86-119. MR531272 (83d:35060)

[26] Lewy, A. "A note on harmonic functions and a hydrodynamical application." Proc. Amer. Math. Soc. 3, (1952): 111-113. MR0049399 (14:168c)

[27] Lighthill, J. Waves in fluids, Cambridge: Cambridge University Press, 1978. MR642980 (84g:76001a) 
[28] Lockhart, R. B. "Fredholm properties of a class of elliptic operators on non-compact manifolds." Duke Math. J. 48, (1981): 289-312. MR610188 (82j:35050)

[29] Matioc, A.-V. "On particle trajectories in linear deep-water waves," to appear in Commun. Pure Appl. Anal.

[30] Matioc, B.-V. "Analyticity of the streamlines for periodic traveling water waves with bounded vorticity." Int. Math. Res. Not. 17, (2011): 3858-3871.

[31] Hur, V. M. "Global bifurcation theory of deep-water waves with vorticity." SIAM J. Math. Anal. 37 , no. 5 (2005): 1482-1521. MR2215274 (2007e:76025)

[32] Triebel, H. Interpolation Theory. Function Spaces. Differential Operators, Heidelberg: Johann Ambrosius Barth Verlag, 1995. MR1328645 (96f:46001) 